\title{
Study on Correlation of Assessment Tools Used to Diagnose Xerostomia
}

\author{
Eun-Hee Lee \\ Department of Dental Hygiene, Choonhae College of Health Sciences, Ulsan, Korea
}

Objective: This study conducted correlation analysis between subjective surveys measuring xerostomia (mouth dryness) and objective assessment methods for measured values of oral moisture. By doing so, it sought to identify the appropriateness of assessment tools to measure xerostomia.

Methods: The study carried out a questionnaire survey among 37 healthy male and female adults without general diseases after getting their consent following the review of IRB (IRB File No.2017-03-035). Measurement of oral moisture was done using Mucus (Life Co., Saitama, Japan). The questionnaire used questions of precedent research related with subjective xerostomia diagnosis. For analysis, the study, using IBM SPSS Statistics version 18.0 (IBM, New York, United States), conducted reliability test by each question, internal consistency and correlation analysis between measured values of oral moisture.

Results: The results of this study are as follows. Cronbach's $\alpha$ value of six questions was 0.840 , showing high reliability between questions. Intraclass correlation coefficient value ranged between 0.40 and 0.75 , showing internal consistency between the questions. 3. As a result of correlation analysis, senses of mouth dryness were statistically significant between the questions $(* p<0.05, * * p<0.01)$, while correlation between measured values of oral moisture and questions were not statistically significant $(* p<0.05)$. As per the findings of this study, there was no correlation between subjective surveys and measured values of oral moisture.

Conclusion: And yet the study believes there is need for diverse research on effectiveness of subjective survey methods and measuring tools in connection with xerostomia.

Keywords: oral dryness, oral humidity, survey

\section{Introduction}

\section{Research background}

\section{Corresponding author Eun-Hee Lee}

E-mail: arch1981@naver.com

(iD) https://orcid.org/0000-0003-1084-8286

Received June 1, 2021, Revised June 15, 2021, Accepted June 26, 2021
Dry mouth is a subjective symptom that makes the inside of the mouth feel dry. It is mainly caused by decreased salivation, changes in the physical properties of saliva, and continuous drug intake due to chronic diseases, head and neck radiation, etc. [1-3]. In modern society, the improvement of living standards and the development of medical technology have increased life expectancy, but the number of the elderly and chronic diseases and the number of people taking drugs to treat them have also increased. Among these therapeutic drugs, many drugs decreased salivation, which eventually re-

Copyright (C) 2021. Korean Academy of Preventive Dentistry. All rights reserved.

This is an Open Access article distributed under the terms of the Creative Commons Attribution Non-Commercial License (http://creativecommons.org/licenses/ by-nc/4.0) which permits unrestricted non-commercial use, distribution, and reproduction in any medium, provided the original work is properly cited. 
sulted in an increase in dry mouth [4-6]. As such, in order to improve the quality of life of patients suffering from dry mouth, there is a need for a method that can accurately determine and treat dry mouth.

In order to evaluate the dry mouth felt by the patient, subjective symptoms and objective signs are evaluated. A questionnaire is mainly used to evaluate a patient's subjective symptoms [7-9]. This is because the evaluation of the subjective symptoms felt by a patient with dry mouth is only possible through a questionnaire.

As an objective evaluation method, diagnosis is made through measurement of salivation rate and clinical evaluation. The decrease in salivation makes the oral mucosa wet by thinning the layer of saliva that wets the oral mucosa, resulting in insufficient wettability of the oral mucosa, leading to the perception of dry mouth. It has been reported that this insufficient wettability of the mucous membrane shows a close correlation with the decrease in the salivation rate [10-12].

Therefore, in diagnosing dry mouth, objective evaluation such as measurement of salivation rate, as well as understanding the degree of symptoms and discomfort subjectively felt by the patient, and understanding the correlation with objective signs are essential for the diagnosis and treatment of dry mouth. it is very important to have.

\section{Purpose of the study}

In this study, the effectiveness of an appropriate evaluation tool in judging dry mouth disease was investigated through correlation analysis between the method of using questionnaire, a subjective evaluation method, and the measurement result of oral humidity related to salivation rate as an objective evaluation tool. We want to help you apply.

Table 1. Demographic characteristics of study subjects

\begin{tabular}{cc}
\hline Variable & $\mathrm{n}$ \\
\hline Gender & \\
Male & 18 \\
Female & 19 \\
Age & \\
$30 \mathrm{~s}$ & 20 \\
$40 \mathrm{~s}$ & 14 \\
$50 \mathrm{~s}$ & 3 \\
Total & 37
\end{tabular}

\section{Materials and Methods}

\section{Research subject}

From April to May 2017, 37 healthy adult men and women who did not complain of dry mouth symptoms, had good oral conditions, had no major systemic diseases, and did not take drugs that could cause dry mouth were selected as subjects. The distribution of subjects by gender and age is as shown in Table 1.

\section{IRB deliberation}

After passing the IRB deliberation (IRB File No.2017-03035), the purpose of this study was explained to the research subjects and consent was obtained.

\section{Measurement of oral humidity}

Oral humidity was measured using Mucus (LifeCo., Saitama, Japan) as shown in (Figure 1). The data are presented as three-digit numbers ranging from 0.00 to 99.8 . According to the manufacturer's instructions, the lingual region was measured three times per session, and the median value was selected as the most reliable and recorded [13]. If the reading standard for oral humidity is less than 28 , it means that the oral cavity is dry, and if it is higher than 28 , it means that the oral cavity is not dry [14]. As a result of oral humidity measurement of all subjects, an average of 30.68 was found without dryness.

\section{Questionnaire evaluation}

As shown in Table 2, the NRS (Numerical Rating Scale) and straight line, which writes the pain and discomfort level felt by the patient in a score table divided at regular numerical intervals [15], based on the questionnaires prepared in previous studies such as this one Subjective dry mouth was eval-

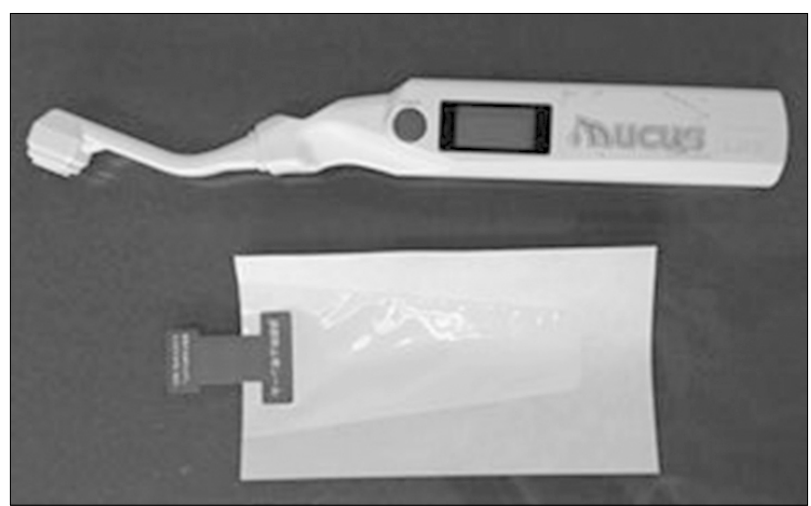

Figure 1. Oral humidity measurement. 
Table 2. 6 questionnaire questions to determine the degree of dry mouth

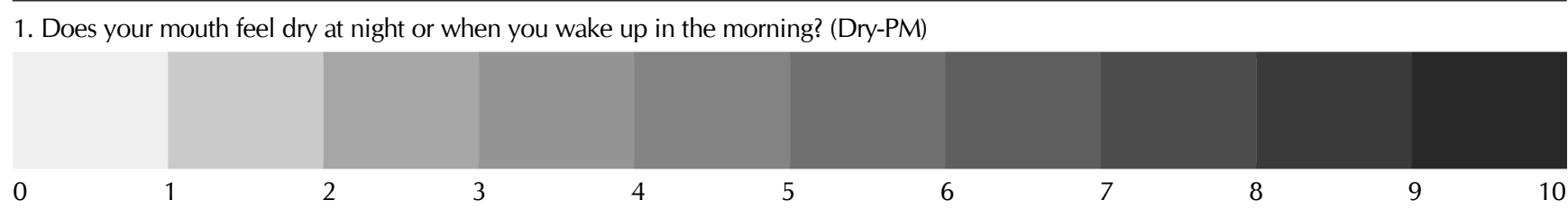

2. Do you usually feel that your mouth is dry during the daytime? (Dry-day)

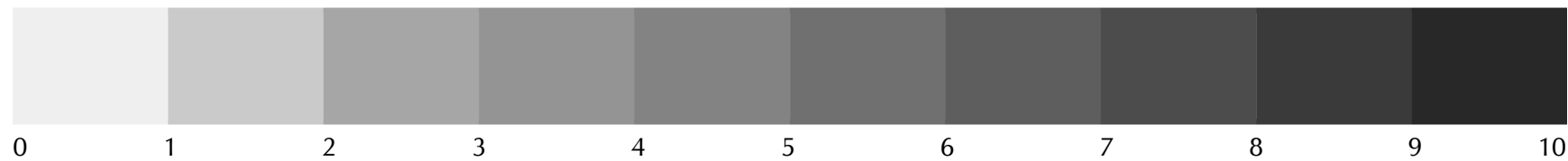

3. Do you usually feel that your mouth is dry during the daytime? (Dry-eat)

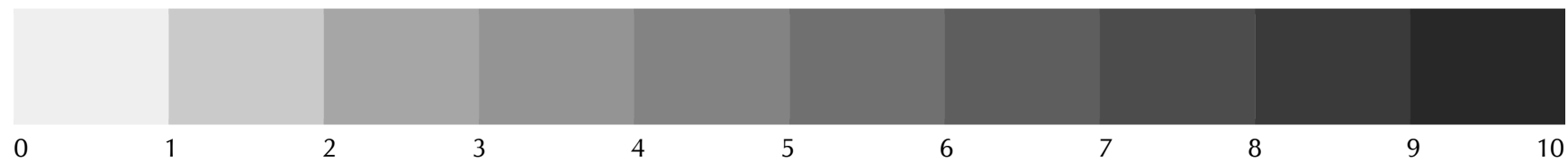

4. Are you having trouble swallowing food because your mouth is dry? (Dif-swal)

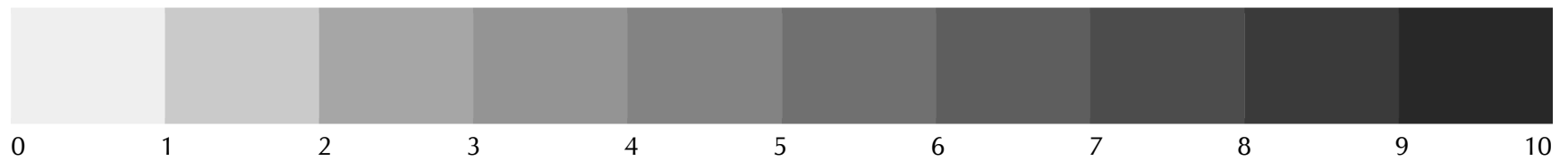

5. Do you feel that the amount of saliva in your mouth is low?(Am-sal)

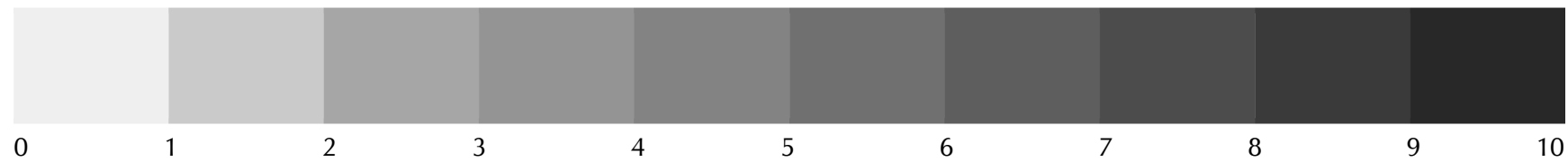

6. If so, to what extent do you feel the discomfort in your daily life due to dry mouth overall? (Eff-life)

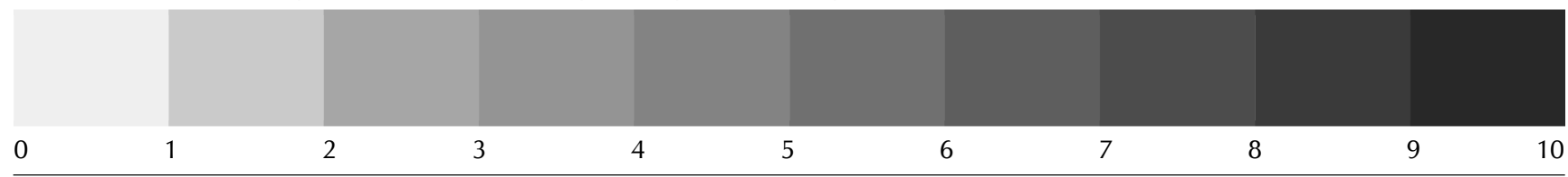

uated by using the form mixed with the Visual Analogue Scale (VAS) shown above.

\section{Data analysis method}

Statistical analysis of measurement results and all collected data was performed using IBM SPSS Statistics version 18 (IBM, Newyork, United States). Reliability evaluation was performed using intraclass correlation coefficient (ICC) obtained by analyzing the result values obtained from six questions asking about the degree of dry mouth. intraclass correlation coefficient.

The value is used to determine the degree of agreement between numerically measured values, and when it is between
0.40 and 0.75 , it can be interpreted as having high reliability in reproducibility, and when it is above 0.75 , it can be interpreted as having very high reliability. In addition, by measuring the Cronbach's $\alpha$ value, it was evaluated whether the six items consistently represent the degree of dry mouth in one structure. Correlation analysis between questionnaire items and oral humidity measurement results was analyzed with Kendall's tau_b after normality test. 


\section{Results}

\section{Reliability evaluation of subjective dry mouth questionnaire}

As a result of examining the reliability of 6 items asking about the degree of dry mouth, as shown in Table 3, the degree of dry mouth during the day (Dry-day) and the degree of dry mouth during meals (Dry-eat) were 0.719 . and 0.727 , indicating high reliability, and the remaining items did not reach 0.75 , but showed relatively high intraclass correlation coefficient values from 0.436 to 0.661 . Cronbach's $\alpha$ value, which evaluates the internal agreement of the six questions, was 0.840 , which was high, indicating that the questionnaire questions had a high internal agreement in indicating the degree of dry mouth.

\section{Correlation analysis of subjective dryness and oral humidity measurement results}

As a result of the normality test, a normal distribution was not achieved, so Kendall's tau_b correlation analysis was performed. The results are shown in Table 4. There was a correlation between the subjective evaluation questionnaire items on dry mouth $(* p<0.05, * * p<0.01)$, but there was no

Table 3. Analysis of reliability and internal consistency between questionnaire item

\begin{tabular}{lccc}
\hline & $\begin{array}{c}\text { Intraclass } \\
\text { correlation } \\
\text { coefficient }\end{array}$ & Significance & Cronbach's $\alpha$ \\
\hline Dry-PM & 0.436 & 0.000 & 0.840 \\
Dry-day & 0.719 & 0.000 & \\
Dry-eat & 0.727 & 0.000 & \\
Dif-swal & 0.626 & 0.000 & \\
Am-sal & 0.611 & 0.000 & \\
Eff-life & 0.661 & 0.000 & \\
\hline
\end{tabular}

clear correlation with the oral humidity measurement result $\left({ }^{*} \mathrm{p}<0.05\right)$.

\section{Conclusion}

For the evaluation of dry mouth, measuring the rate of salivation has been used as the most objective and general test. When salivation is reduced, not only dry mouth, but also pain such as burning sensation due to dry mouth, changes in taste, and difficulty in swallowing or eating dry food may be complained of [16]. However, there are patients who complain of dry mouth even though they do not show a clear decrease in salivation, and it has also been reported that the depth of subjective dry mouth does not have a direct correlation with the decrease in salivation rate [17-19]. In addition, the salivation rate, residual saliva volume in the oral cavity, $\mathrm{pH}$, etc. do not necessarily correlate with the onset of dry mouth and discomfort $[20,21]$. Therefore, this study was conducted to help provide an accurate diagnosis and appropriate treatment by analyzing the correlation between evaluation tools that have proven reliability and validity in diagnosing dry mouth. As a result of comparing the questionnaire, which is a subjective evaluation tool, and oral humidity measurement, an objective evaluation tool, the reliability between the items was relatively high at 0.840 , and the internal consistency was between 0.40 and 0.75 . As a result of correlation analysis between evaluation tools, correlations were found for each item $(* p<0.05$, $* * \mathrm{p}<0.01$ ), but there was no correlation between the questionnaire and oral humidity measurements $(* p<0.05)$.

It is very important to verify the reliability and validity of the evaluation tool used in diagnosing dry mouth. Although various studies on various evaluation tools have been conducted in previous studies, it is still difficult to apply an appropriate evaluation tool to diagnose dry mouth in clinical practice. As a result of measuring and comparing oral humidity related to salivation with a questionnaire, which is a subjective evaluation tool for dry mouth, and an objective evalua-

Table 4. Correlation between oral humidity and dryness $(N=37)$

\begin{tabular}{|c|c|c|c|c|c|c|c|}
\hline & Oral humidity & Dry-PM & Dry-day & Dry-eat & Dif-swal & Am-sal & Eff-life \\
\hline Oral humidity & 1 & & & & & & \\
\hline Dry-PM & 0.060 & 1 & & & & & \\
\hline Dry-day & -0.020 & $0.497 * *$ & 1 & & & & \\
\hline Dry-eat & -0.015 & 0.186 & $0.538^{* *}$ & 1 & & & \\
\hline Dif-swal & 0.045 & 0.197 & $0.269 *$ & $0.492 * *$ & 1 & & \\
\hline Am-sal & 0.037 & 0.205 & $0.433^{* *}$ & $0.524 * *$ & $0.515^{* *}$ & 1 & \\
\hline Eff-life & -0.165 & $0.332 * *$ & $0.460 * *$ & $0.506^{* *}$ & $0.391 * *$ & $0.492 * *$ & 1 \\
\hline
\end{tabular}

${ }^{*} \mathrm{p}<0.05, * * \mathrm{p}<0.01$ 
tion method, there was no correlation between the measurement tools. In the future, it is thought that a study on an accurate diagnosis and appropriate coping method according to the symptom level and subjective feeling of dry mouth is necessary.

\section{Conflict of Interest}

No potential conflict of interest relevant to this article was reported.

\section{ORCID}

Eun-Hee Lee, https://orcid.org/0000-0003-1084-8286

\section{References}

1. Fox PC, van der Ven PF, Sonies BC, Weiffenbach JM, Baum BJ. Xerostomia: evaluation of a symptom with increasing significance. J Am Dent Assoc 1985;110:519-25.

2. Atkinson JC, Wu A. Salivary gland dysfunction: causes, symptoms, treatment. J Am Dent Assoc 1994;125:409-16.

3. Guggenheimer J, Moore PA. Xerostomia: etiology, recognition and treatment. J Am Dent Assoc 2003;134:61-9.

4. Wu AJ, Ship JA. A characterization of major salivary gland flow rates in the presence of medications and systemic diseases. Oral Surg Oral Med Oral Pathol 1993;76:301-06.

5. Narhi T, Meurman J, Ainamo A, et al. Association between salivary flow rate and the use of systemic medication among 76-, 81-, and 86-year-old inhabitants in Helsinki, Finland. J Dent Res 1992;71:1875-80.

6. Schein OD, Hochberg MC, Munoz B, et al. Dry eye and dry mouth in the elderly: a population-based assessment. Arch Intern Med 1999;159:1359-63.

7. Fox PC, Busch K, Baum BJ. Subjective reports ofxerostomia and objective measures of salivary gland performance. J Am Dent Assoc 1987;115:581-84.

8. Nederfors T. Xerostomia: Prevalence and pharmacotherapy. With special reference to -adrenoreceptor antagonists. Swed Dent J 1996;Suppl.116:1-70.
9. Thomson WM, Chalmers JM, Spencers AJ, Ketabi M. The occurrence of xerostmia and salivary gland hypofunction in a population-based sample of older South Australians. Spec Care Dent 1999;19:20-3.

10. DiSabato-Mordarski T, Kleinberg I. Measurement and comparison of the residual saliva on various oral mucosal and dentition surfaces in humans. Arch Oral Biol 1996;41:655-65.

11. Wolff M, Kleinberg I. Oral mucosal wetness in hypoand normosalivators. Arch Oral Biol 1998;43:455-62.

12. Lee SK, Lee SW, Chung SC, Kim YK, Kho HS. Analysis of residual saliva and minor salivary gland secretions in patients with dry mouth. Arch Oral Biol 2002;47:637-41.

13. IshimotoS,TsunodaK,FujimakiY,OkadaK,SaitoY,KinoshitaM ,etal.Objective andnon -invasiveevaluationofdrymouth.Auris NasusLarynx2008;35:89-93.

14. YamadaH,NakagawaY,NomuraY,YamamotoK,SuzukiM,Wata nabeNY,etal. Preliminary results of moisture checker for Mucus in diagnosing dry mouth. Oral Dis2005;11:405-7.

15. Lee, J. Y., Lee, Y. O., \& Kho, H. S. Reliability of a questionnaire for evaluation of dry mouth symptoms. Journal of Oral Medicine and Pain, 2005:30.

16. Loesche WJ, Bromberg J, Terpenning MS, et al. Xerostomia, xerostomic medications and food avoidances in selected geriatric groups. J Am Geriatr Soc 1995;43:401-07.

17. Spielman A, Ben-Aryeh H, Gutman D, Szargel R, Deutsch E. Xerostomia diagnosis and treatment. Oral Surg Oral Med Oral Pathol 1981;51:144-7.

18. von Knorring L, Mornstad H. Qualitative changes in saliva composition after short-term administration of imipramine and zimelidine in healthy volunteers. Scand J Dent Res 1981;89: 313-20.

19. Donatsky O, Johnsen T, Holmstrup P, Bertram U. Effect of "Saliment" on parotid salivary gland secretion and on xerostomia caused by Sjogren's syndrome. Scand J Dent Res 1982; 90:157-62

20. Villa A, Abati S. Risk factors and symptoms associated with xerostomia: a cross-sectional study. Aust Dent J 2011;56:290-5.

21. Pai S, Ghezzi EM, Ship JA. Development of a Visual Analogue Scale questionnaire for subjective assessment of salivary dysfunction. Oral Surg Oral Med Oral Pathol Oral Radiol Endod 2001;91:311-6. 